\title{
Publishing Formal Specifications in Z Notation on World Wide Web
}

\author{
Luboš Mikušiak \\ Intergraph SR \\ Matejkova 20,841 05 Bratislava, Slovak Republic \\ E-mail: Imikusia@ingr.com \\ Miroslav Adámy \\ Department of Computer Science and Software Engineering \\ Faculty of Electrical Engineering and Information Technology \\ Ilkovicova 3, 81219 Bratislava, Slovak Republic \\ E-mail: adamy@elf.stuba.sk \\ Thomas Seidmann \\ Department of Computer Science and Software Engineering \\ Faculty of Electrical Engineering and Information Technology \\ Ilkovicova 3. 81219 Bratisiava. Slovak Republic \\ E-mail: seidmann@dcs.elf.stuba.sk
}

\begin{abstract}
This article presents $Z$ Browser Plug-in, Netscape Navigator plug-in and ActiveX control which enables the usage of WWW clients for viewing HTML pages with embedded LaTeX documents containing $Z$ specifications.
\end{abstract}

\section{Keywords:}

formal specification, $Z$ notation, Netscape plug-in; ActiveX control, WWW, LaTeX

\section{Introduction}

The formal specification notation Z (pronounced "zed") is based on set theory and first order predicate logic. It has been developed by the Programming Research Group (PRG) at the Oxford University Computing Laboratory (OUCL) and elsewhere since the late 1970s, inspired by Jean-Raymond Abrial's seminal work.

$Z$ is now used by industry as part of the software (and hardware) development process in both Europe and the US. It is currently undergoing international ISO standardization. 
It has been previously difficult to display properly $Z$ specifications in World Wide Web browsers. There were problems displaying schema boxes and many symbols of $Z$ like maplet, relational image, bag and sequence display and many others. Currently, there is a work in progress to add mathematical extensions to HTML + including support for $Z$.

There has been many documents written in LaTeX using fuzz.sty or zed.sty styles, or in the compatible styles oz.sty and mz:sty. The number of LaTeX documents will be growing also because most of the $Z$ type checkers like fuzz [2] and ZTC type checker [3], and theorem provers like Z/EVES [4] use this format as an input format. Some other tools like Formaliser [5] and CADiZ [7] are able to process $\mathrm{Z}$ specifications in LaTeX. Many books on $\mathrm{Z}$ including The $Z$ Notation A Reference Manual by Mike Spivey [1] were prepared using LaTeX.

This article describes $Z$ Browser Plug-in - a Netscape plug-in and ActiveX control, which are able to display $Z$ specifications in LaTeX as embedded objects of HTML pages. Displayed $Z$ paragraphs are seamlessly integrated with the rest of the HTML page and they appear in the same form as when printed by LaTeX. In order to be displayed, the LaTeX documents do not need to be modified or pre-processed.

\section{About the $Z$ Notation}

The formal specification in $\mathrm{Z}$ is decomposed into small pieces called schemas [1]. By splitting the specification into schemas, it can be presented piece by piece. Each piece can be linked with a commentary which explains informally the significance of the formal mathematics. In $\mathrm{Z}$, schemas are used to describe both static and dynamic aspects of the system.

The most representative summary of applications of $Z$ in industrial projects can be found in [8]. Another overview can be found in [9]. Among other applications, $Z$ was used for specification of the IEEE Floating Point Standard, a scheduler for the T-800 Transputer, for respecification of IBM's Customer Information Control System and for specification of the Airbus A330/340 cabin illumination system.

\section{Z Browser Plug-in}

Plug-ins are software modules that are seamlessly integrated into Navigator, appearing simply as supplemental capabilities [10]. Z Browser Plug-in is a Microsoft Windows dynamic link library (DLL) which acts as a Netscape plug-in in Microsoft Windows. In can process and display embedded LaTeX documents. Z Browser Plug-in further recognizes following attribute used with the EMBED tag, which further specify which portion of the LaTeX document will be displayed:

- PARAG_NUMBER="paragr_number" defines what $Z$ paragraph of the LaTeX document will be displayed. 
Here is a small example of a HTML file with two embedded LaTeX documents:

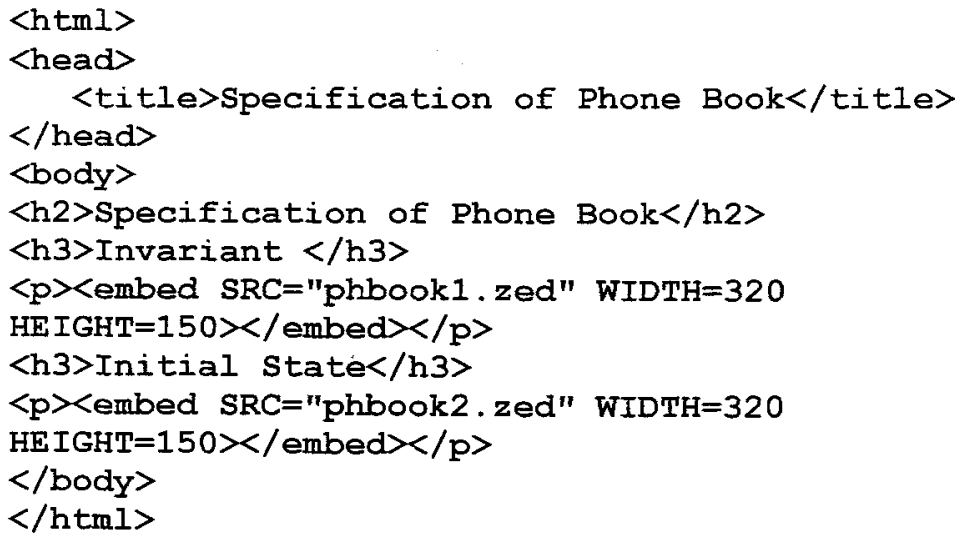

$Z$ Browser Plug-in is able to display symbols of the $Z$ notation in different color and when user clicks by mouse on such a symbol once, short description of the symbol is displayed in the status bar of Netscape. After a double-click, help topic for selected symbols is displayed in Windows Help utility. The help file for the $Z$ Notation is identical with the one provided with $Z$ Browser [6].

\section{Z Browser ActiveX Control}

ActiveX is just another term for COM/OLE based technology from Microsoft Inc. It is currently available under (albeit not limited to) the MS Windows operating systems. MS Internet Explorer supports objects according to the HTML 3.2 object model. Objects add functionality to HTML document by letting you insert images, video, and programs, such as JAVA applets, and ActiveX controls. To insert an ActiveX control you use the OBJECT element, supplying attribute values that specify the object type, location, initial data, and so on. If the object has configurable properties, you can set these using the PARAM element. The following example shows how to insert the $Z$ Browser ActiveX control and fill it with content:

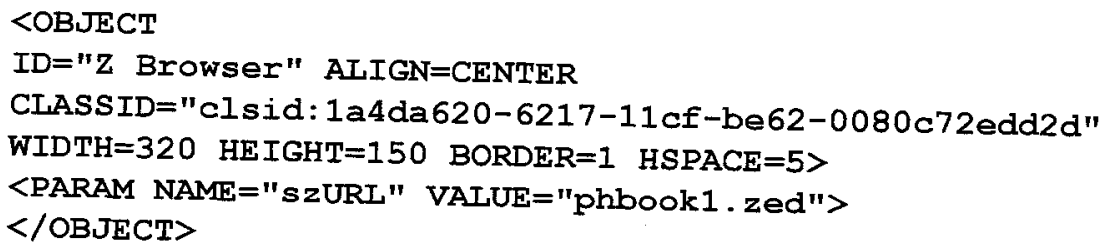

If the control uniquely identified by its CLASSID is not yet installed and registered on the client's workstation, it gets downloaded from the specified location (the specification is not included in this example), registered and executed after 
authentication (signature check). Note that the actual value of CLSID is just an example of a GUID.

\section{Conclusions}

Making Z specifications easily available on World Wide Web is an important action in order to popularize this formal notation and its benefits among the rapidly growing number of Internet users. Easy access to the HTML pages with $Z$ specifications via links from other HTML pages can bring into contact with $Z$ more users than ever before. Having the interactive help functionality, users can learn basics of $Z$ just by viewing HTML pages with $Z$ specifications and reading appropriate help topics.

Many researchers will appreciate the possibility to publish their $Z$ specifications on World Wide Web. This possibility was missing before, and so either LaTeX documents had to be e-mailed to those who were interested or the hard copies had to be sent.

\section{References}

1. Spivey J. M.: The Z Notation A Reference Manual, Prentice Hall, 1989

2. Spivey J. M.: The fuzz Manual, Computing Science Consultancy, 1992

3. Xiaoping Jia: ZTC: A Type Checker for Z -- User's Guide, Institute for Software Engineering, Department of Computer Science and Information Systems, DePaul University, 1992

4. Saaltink M.: $Z$ and EVES, Z User Workshop, Proceedings of the 4th Annual Z User Meeting, 1990

5. Flynn M., Hoverd T., Braizer D.: Formaliser - An Interactive Support Tool for Z, Z User Workshop, Proceedings of the 4th Annual Z User Meeting, 1990

6. Mikusiak L. et al.: Z Browser - Tool for Visualisation of $Z$ Specifications, ZUM'95 - 9th International Conference of Z Users, Springer-Verlag, 1995

7. Toyn I.: CADiZ Quick Reference Guide, York Software Engineering Ltd, 1990

8. Hinchey M., Bowen J.: Applications of Formal Methods, Prentice Hall International Series in Computer Science, 1995

9. Craigen, D., Gerhart S., and Ralston T., An International Survey of Industrial Applications of Formal Methods, Volume 1 Purpose, Approach, Analysis and Conclusions; Volume 2 Case Studies, U.S. National Institute of Standards and Technology, GCR 93/626, March 1993

10. Netscape Navigator LIVE CONNECT / PLUG-IN Software Develpment Kit, $\mathrm{http}: / /$ home.netscape.com/comprod/development_partners/plugin_api

11. Learn about Internet Explorer, http://www.microsoft.com/ie

12. The ActiveX Working Group, http://www.activex.org

13. The ActiveX Software Development Kit, Microsoft Corp. 